\title{
BiBLIOGRAPHY
}

1. Willard E. Baxter, Lie simplicity of a special class of associative ring, Proc. Amer. Math. Soc. vol. 7 (1956) pp. 855-863.

2. I. N. Herstein, On the Lie and Jordan rings of a simple associative ring, Amer. J. Nath. vol. 77 (1955) pp. 279-285. 575 .

3. - - On the Lie ring of a division ring, Ann. of Math. vol. 60 (1954) pp. 571-

4. - The Lie ring of a simple associative ring, Duke Math. J. vol. 22 (1955) pp. $471-476$.

Louisiana State University

\section{RATIONAL APPROXIMATION TO SOLUTIONS OF ALGEBRAIC DIFFERENTIAL EQUATIONS}

E. R. KOLCHIN ${ }^{1}$

Introduction. It was observed by Liouville (C. R. Acad. Sci. Paris, vol. 18 (1844) pp. 910-911; J. Math. Pures Appl. vol. 16 (1851) pp. 133-142) that an element $\alpha$ of the field $C$ of complex numbers which is algebraic of degree $n$ (over the ring $\boldsymbol{Z}$ of rational integers) can not be approximated very well by rational numbers, in the following sense: there exists a real number $\gamma>0$ such that $|\alpha-p / q| \geqq \gamma /|q|^{n}$ for all $p, q \in \boldsymbol{Z}$ with $q \neq 0$ and $p / q \neq \alpha$. Using this theorem Liouville gave the first examples of transcendental numbers. The proof depends only on the circumstance that every nonzero element of $\boldsymbol{Z}$ has absolute value $\geqq 1$ and the following two obvious facts (in the statement of which $f$ denotes the polynomial of degree $n$ vanishing at $\alpha$ ): (i) $\alpha$ is an isolated point of the set of zeros of $f$; (ii) $f(y / z)$ can be written as a fraction in which the numerator is a polynomial in $y$ and $z$ and the denominator is $z^{n}$. It follows that Liouville's theorem has an abstract version in which $\boldsymbol{C}$ and $\boldsymbol{Z}$ are replaced by an arbitrary nontrivially valued field and a nonzero subring thereof in which each nonzero element has value $\geqq 1$. Since the field $K\left(\left(X^{-1}\right)\right)$ of power series in the reciprocal of an indeterminate $X$ over a given commutative field $K$ admits a valuation for which the series $u=c_{m} X^{-m}$ $+c_{m+1} X^{-(m+1)}+\cdots\left(\right.$ with $\left.c_{m} \neq 0\right)$ has the value $|u|=e^{-m}$, and the polynomial ring $K[X]$ is a subring of $K\left(\left(X^{-1}\right)\right)$ in which every nonzero element has value $\geqq 1$, Liouville's theorem applies in this situa-

Received by the editors July 25, 1958.

1 This paper was prepared in connection with a grant from the National Science Foundation. 
tion, that is, his theorem is also a result on the approximation of algebraic functions by rational ones.

An algebraic equation may be considered as an algebraic differential equation of order zero, so that algebraic functions are special cases of differentially algebraic ones. The purpose of this note is to extend Liouville's theorem (abstract version) to an ordinary differential field with valuation which is subject to a certain not unnatural condition. A weaker and slightly less general result for the special case of power series has been obtained by E. Maillet (Nombres transcendents, Paris, 1906). The present treatment is, I believe, more transparent.

The condition on the valuation, which can not be omitted, and which in the case of $K\left(\left(X^{-1}\right)\right)$ is equivalent to the condition that the field characteristic be 0 , is used in $\$ 1$ to define "valued differential field." The fact (i) above is suitably generalized in $\$ 3$. Since a differential polynomial in general has infinitely many solutions, the generalization is not quite obvious; it is proved using a lemma on wronskian determinants established in $\$ 2$. The generalization of fact (ii) presents no difficulty provided the exponent $n(=\operatorname{deg} f)$ is replaced by a generally bigger number called the "denomination" of $f$. When the differential polynomial $f$ is of the order 0 , its denomination coincides with its degree. This (and a slight generalization thereof) is described in $\$ 4$. The proof of the approximation theorem itself, which is then trivial, is found in $\$ 5$. An almost immediate consequence of the theorem is that a power series $\sum_{k=0}^{\infty} c_{k} X^{s_{k}}$, with nonzero coefficients $c_{k}$ in a field $K$ of characteristic 0 and with strictly increasing integral exponents $s_{k}>0$ such that the sequence of ratios $s_{k+1} / s_{k}$ is unbounded, is differentially transcendental over $K(X)$.

It remains to make the obvious remark, in view of the deep ThueSiegel-Roth improvement of Liouville's theorem (see K. F. Roth, Mathematika vol. 2 (1955) pp. 1-20), that it would be desirable to obtain a similar improvement in the present theorem.

Terminology and notation. In what follows, all differential rings and fields are ordinary and commutative; the derivative of an element $a$ of a differential ring or field is denoted by $a^{\prime}$, and for each natural number $k$ the $k$ th derivative of $a$ is denoted by $a^{(k)}$. All valuations are nonarchimedean; the value group of any valuation is written multiplicatively, and the value of an element $a$ is denoted by $|a|$.

\section{Valued differential fields.}

Definition. A valued differential field is a differential field endowed with a valuation satisfying the following condition: there exist elements $\alpha$ and $\beta$ of the value group such that 


$$
\alpha|a| \leqq\left|a^{\prime}\right| \leqq \beta|a|
$$

for every element $a$ of the differential field with $|a|<1$. The elements $\alpha$ and $\beta$ are then called, respectively, lower and upper bounds of the valued differential field. The valued differential field is said to be trivial or nontrivial according as the value group is trivial or not.

Lemma 1. If $\mathfrak{F}$ is a nontrivial valued differential field with lower bound $\alpha$ and upper bound $\beta$, then the second inequality in (1) holds for every $a \in \mathcal{F}$, and the first inequality in (1) holds for every $a \in F$ with $|a| \neq 1$.

Proof. When $|a|<1$ then (1) holds by hypothesis. When $|a|>1$ then $a \neq 0$ and $\left|a^{-1}\right|<1$; since $\left|a^{\prime} / a\right|=\left|\left(a^{-1}\right)^{\prime} / a^{-1}\right|$, again (1) holds. It remains to prove the second part of (1) when $|a|=1$. By hypothesis there exists a nonzero element $b \in \mathcal{F}$ with $|b| \neq 1$, whence $|a b| \neq 1$, and therefore $\left|b^{\prime} / b\right| \leqq \beta$ and $\left|a^{\prime} / a+b^{\prime} / b\right|=\left|(a b)^{\prime} / a b\right| \leqq \beta$; if $\left|a^{\prime} / a\right|$ $\leqq\left|b^{\prime} / b\right|$ then obviously $\left|a^{\prime} / a\right| \leqq \beta$, whereas if $\left|a^{\prime} / a\right|>\left|b^{\prime} / b\right|$ then $\left|a^{\prime} / a\right|=\left|a^{\prime} / a+b^{\prime} / b\right| \leqq \beta$.

Corollary 1. Hypothesis as in Lemma 1 , let $n$ be a natural number. Then $\left|a^{(n)}\right| \leqq \beta^{n}|a|$ for every $a \in \mathcal{F}$, and $\left|a^{(n)}\right| \geqq \alpha^{n}|a|$ for every $a \in \mathcal{F}$ with $|a|>1$ and $|a|>1 / \alpha^{n-1}$.

Proof. Induction on $n$.

COROllary 2. Let $\mathcal{F}$ be a nontrivial valued differential field. Every nonzero constant $c$ in $\mathcal{F}$ has value $|c|=1$. The characteristic of $\mathcal{F}$ is 0 .

PROOF. If there existed a nonzero constant $c$ with $|c| \neq 1$ we would have $0=|0|=\left|c^{\prime}\right| \geqq \alpha|c|>0$. If the characteristic were $p \neq 0$ then, for every nonzero $a \in \mathcal{F}, a^{p}$ would be a nonzero constant, we would have $|a| p=\left|a^{p}\right|=1$, and the valuation would be trivial.

2. Wronskian determinants. If $\left(a_{1}, \cdots, a_{n}\right)$ is a finite sequence of elements of a differential ring with unity element, we denote the wronskian determinant $\operatorname{det}\left(a_{j}^{(i-1)}\right)_{1 \leq i \leq n, 1 \leq j \leq n}$ by $W\left(a_{1}, \cdots, a_{n}\right)$. The wronskian determinant equals 1 when $n=0$ and equals $a_{1}$ when $n=1$. If $n>0$ and we expand the determinant by the minors of the last row, we obtain the formula

$$
\sum_{j=1}^{n}(-1)^{n+j} a_{j}^{(n-1)} W\left(a_{1}, \cdots, a_{j-1}, a_{j+1}, \cdots, a_{n}\right)=W\left(a_{1}, \cdots, a_{n}\right) .
$$

Applying the same device to the determinant obtained on replacing the last row by one of the other rows, we obtain the formula 


$$
\begin{aligned}
\sum_{j=1}^{n}(-1)^{n+j} a_{j}^{(i)} W\left(a_{1}, \cdots, a_{j-1}, a_{j+1}, \cdots, a_{n}\right) & =0 \\
& (0 \leqq i \leqq n-2) .
\end{aligned}
$$

Lemma 2. If $b, a_{1}, \cdots, a_{n}$ are elements of a differential ring with unity element then $W\left(b a_{1}, \cdots, b a_{n}\right)=b^{n} W\left(a_{1}, \cdots, a_{n}\right)$.

Proof. If $n=0$ or 1 this is obvious. Let $n>1$ and suppose the result known for lower values of $n$. Then, by (2) and (3), $W\left(b a_{1}, \cdots, b a_{n}\right)$

$$
\begin{aligned}
& =\sum_{j=1}^{n}(-1)^{n+j}\left(b a_{j}\right)^{(n-1)} W\left(b a_{1}, \cdots, b a_{j-1}, b a_{j+1}, \cdots, b a_{n}\right) \\
& =\sum_{j=1}^{n}(-1)^{n+j} \sum_{i=0}^{n-1}\left(\begin{array}{c}
n-1 \\
i
\end{array}\right) b^{(n-1-i)} a_{j}^{(i)} b^{n-1} W\left(a_{1}, \cdots, a_{j-1}, a_{j+1}, \cdots, a_{n}\right) \\
& =b^{n-1} \sum_{i=0}^{n-1}\left(\begin{array}{c}
n-1 \\
i
\end{array}\right) b^{(n-1-i)} \sum_{j=1}^{n}(-1)^{n+j} a_{j}^{(i)} W\left(a_{1}, \cdots, a_{j-1}, a_{j+1}, \cdots, a_{n}\right) \\
& =b^{n-1} b W\left(a_{1}, \cdots, a_{n}\right)=b^{n} W\left(a_{1}, \cdots, a_{n}\right) .
\end{aligned}
$$

Lemma 3. Let $a_{1}, \cdots, a_{n}$ be elements of a nontrivial valued field with lower bound $\alpha$ and upper bound $\beta$.

(a) $\left|W\left(a_{1}, \cdots, a_{n}\right)\right| \leqq \beta^{n(n-1) / 2}\left|a_{1} \cdots a_{n}\right|$.

(b) If $\left|a_{j}\right|<(\alpha / \beta)^{j-2}\left|a_{j-1}\right|(2 \leqq j \leqq n)$ then $\left|W\left(a_{1}, \cdots, a_{n}\right)\right|$ $\geqq \alpha^{n(n-1) / 2}\left|a_{1} \cdots a_{n}\right|$.

REMARK. The condition in part (b) can be weakened.

Proof. The lemma is obvious if $n=0$ or 1 , and also if $a_{j}=0$ for some $j$. Let $n>1$ and $a_{j} \neq 0$ for every $j$, and suppose the lemma proved for lower values of $n$. By Lemma 2,

$$
\begin{aligned}
W\left(a_{1}, \cdots, a_{n}\right) & =a_{1}^{n} W\left(1, a_{2} / a_{1}, \cdots, a_{n} / a_{1}\right) \\
& =a_{1}^{n} W\left(\left(a_{2} / a_{1}\right)^{\prime}, \cdots,\left(a_{n} / a_{1}\right)^{\prime}\right) .
\end{aligned}
$$

Then

$$
\begin{aligned}
\left|W\left(a_{1}, \cdots, a_{n}\right)\right| & \leqq\left|a_{1}\right|{ }^{n} \beta^{(n-1)(n-2) / 2}\left|\left(a_{2} / a_{1}\right)^{\prime} \cdots\left(a_{n} / a_{1}\right)^{\prime}\right| \\
& \leqq\left|a_{1}\right|{ }^{n} \beta^{(n-1)(n-2) / 2} \beta\left|a_{2} / a_{1}\right| \cdots \beta\left|a_{n} / a_{1}\right| \\
& =\beta^{n(n-1) / 2}\left|a_{1} \cdots a_{n}\right| .
\end{aligned}
$$

Also, under the condition of part (b), 


$$
\begin{aligned}
\left|\left(a_{j+1} / a_{1}\right)^{\prime}\right| & \leqq \beta\left|a_{j+1} / a_{1}\right|<\beta(\alpha / \beta)^{j-1}\left|a_{j} / a_{1}\right|=(\alpha / \beta)^{j-2} \alpha\left|a_{j} / a_{1}\right| \\
& \leqq(\alpha / \beta)^{j-2}\left|\left(a_{j} / a_{1}\right)^{\prime}\right|(2 \leqq j \leqq n-1),
\end{aligned}
$$

so that

$$
\begin{aligned}
\left|W\left(a_{1}, \cdots, a_{n}\right)\right| & \geqq\left|a_{1}\right|{ }^{n} \alpha^{(n-1)(n-2) / 2}\left|\left(a_{2} / a_{1}\right)^{\prime}\right| \cdots\left|\left(a_{n} / a_{1}\right)^{\prime}\right| \\
& \geqq\left|a_{1}\right|{ }^{n} \alpha^{(n-1)(n-2) / 2} \alpha\left|a_{2} / a_{1}\right| \cdots \alpha\left|a_{n} / a_{1}\right| \\
& =\alpha^{n(n-1) / 2}\left|a_{1} \cdots a_{n}\right| .
\end{aligned}
$$

3. Simple zeros. Let $P$ be an element of the differential polynomial ring in a differential indeterminate $y$ over a differential field. A zero of $P$ which fails to be a zero of $\partial P / \partial y^{(i)}$ for at least one natural number $i$ is said to be simple.

Lemma 4. Let $\mathcal{F}$ be a nontrivial valued differential field, and let $u \in \mathcal{F}$ be a simple zero of a differential polynimial $P \in \mathcal{F}\{y\}$. Then there exists an element $\gamma_{0}$ of the value group such that $|v-u| \geqq \gamma_{0}$ for every $v \in \mathcal{F}$ which is a zero of $P$ different from $u$.

Proof. Translating by $u$, we may suppose that $u=0$. Then $P(0)=0$, and there is a natural number $i$ such that $\left(\partial P / \partial y^{(i)}\right)(0) \neq 0$. Let $n$ denote the biggest such $i$. Then we may write $P=L+R$, where

$$
L=\sum_{i=0}^{n} b_{i} y^{(i)}, \quad b_{i} \in \mathcal{F}(0 \leqq i \leqq n), \quad b_{n} \neq 0
$$

and

$$
R=\sum_{k} c_{k} y^{\left(i_{k 1}\right)} \cdots y^{\left(i_{k, h(k)}\right)}, \quad c_{k} \in \mathcal{F}, \quad h(k) \geqq 2(\text { all } k) .
$$

For any zero $v \in \mathcal{F}$ of $P$ with $|v| \leqq 1$,

$$
\begin{aligned}
|L(v)| & =\left|\sum_{k} c_{k} v^{\left(i_{k 1}\right)} \cdots v^{\left(i_{k, h(k)}\right)}\right| \\
& \leqq \sup _{\boldsymbol{k}}\left|c_{k}\right|\left|v^{\left(i_{k 1}\right)}\right| \cdots\left|v^{\left(i_{k, h(k)}\right)}\right| \\
& \leqq \sup _{\boldsymbol{k}}\left|c_{k}\right| \beta^{i_{k 1}+\cdots+i_{k, h(k)}}|v|^{h(k)} \\
& \leqq \gamma_{1}|v|^{2}
\end{aligned}
$$

where $\gamma_{1}=\sup _{k}\left|c_{k}\right| \beta^{i_{k 1}+\cdots+i_{k, h(k)}}$ is independent of $v$.

Now assume the lemma false. Then $\mathcal{F}$ contains zeros $v_{1}, \cdots, v_{n+1}$ of $P$, distinct from 0 , such that 


$$
\begin{aligned}
&\left|v_{1}\right|<1, \quad\left|v_{1}\right|<\gamma_{1}^{-1} \alpha^{n}(\alpha / \beta)^{n(n-1) / 2}\left|b_{n}\right| \\
&\left|v_{j}\right|<(\beta / \alpha)^{j-2}\left|v_{j-1}\right|(2 \leqq j \leqq n+1) .
\end{aligned}
$$

By the above, $\left|L\left(v_{j}\right)\right| \leqq \gamma_{1}\left|v_{j}\right|^{2}(1 \leqq j \leqq n+1)$. By the definition of $L$,

$$
\sum_{i=0}^{n} b_{i} v_{j}^{(i)}=L\left(v_{j}\right) \quad(1 \leqq j \leqq n+1) .
$$

Considering this as a system of linear equations for $b_{0}, b_{1}, \cdots, b_{n}$, and solving for $b_{n}$, we find that

$$
W\left(v_{1}, \cdots, v_{n+1}\right) b_{n}=\sum_{j=1}^{n+1}(-1)^{n+1+j} W\left(v_{1}, \cdots, v_{j-1}, v_{j}, \cdots, v_{n+1}\right) L\left(v_{j}\right) .
$$

It follows by Lemma 3 that

$$
\begin{aligned}
\alpha^{n(n+1) / 2} & \left|v_{1} \cdots v_{n+1}\right|\left|b_{n}\right| \leqq\left|W\left(v_{1}, \cdots, v_{n+1}\right) b_{n}\right| \\
& \leqq \sup _{j}\left|W\left(v_{1}, \cdots, v_{j-1}, v_{j+1}, \cdots, v_{n+1}\right) L\left(v_{j}\right)\right| \\
& \leqq \sup _{j} \beta^{n(n-1) / 2}\left|v_{1} \cdots v_{j-1} v_{j+1} \cdots v_{n+1}\right| \gamma_{1}\left|v_{j}\right|^{2} \\
& =\sup _{j} \beta^{n(n-1) / 2} \gamma_{1}\left|v_{1} \cdots v_{n+1}\right|\left|v_{j}\right| \\
& =\beta^{n(n-1) / 2} \gamma_{1}\left|v_{1} \cdots v_{n+1}\right|\left|v_{1}\right|,
\end{aligned}
$$

so that $\left|v_{1}\right| \geqq \gamma_{1}^{-1} \alpha^{n}(\alpha / \beta)^{n(n-1) / 2}\left|b_{n}\right|$. This contradicts the definition of $v_{1}$ and completes the proof.

4. Denomination. A nonzero differential polynomial $P$ in a differential indeterminate $y$ is a linear combination with nonzero coefficients of certain monomials $y^{e_{0}} y^{\prime e_{1}} \cdots y^{(k) e k} \ldots$. The biggest of the natural numbers $\sum_{k}(k+1) e_{k}$ will be called the denomination of $P$. If $z$ is another differential indeterminate, and we denote the denomination of $P$ by $d$, then $P(y / z)$ can be written with denominator $z^{d}$, that is, $z^{d} P(y / z)$ is a differential polynomial in $y$ and $z$.

More generally, for each natural number $s \geqq 1$, the biggest of the natural numbers $\sum_{k}(k+s) e_{k}$ will be called the $s$-denomination of $P$. (The 1-denomination of $P$ is thus its denomination.) If we denote the $s$-denomination of $P$ by $d_{s}$ then $P\left(y / z^{s}\right)$ can be written with de-

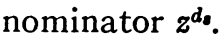

Now let $\mathcal{F}$ be a differential field, let $\mathcal{Z}$ be a differential subring of $\mathcal{F}$, and let $u$ be an element of $\mathcal{F}$ which is differentially algebraic over $\mathcal{Z}$. The smallest natural number $d$ such that there exists a nonzero differential polynomial in $Z\{y\}$ having denomination $d$ and vanishing at $u$ 
will be called the denomination of $u$ over $\mathrm{Z}$. The definition of the $s$-denomination of $u$ over $\mathrm{Z}$ is similar.

Suppose now that the characteristic of $\mathcal{F}$ is zero.

If $P \in Z\{y\}$ vanishes at $u$ and has $s$-denomination equal to that of $u$ over $\mathcal{Z}$, then $u$ is a simple zero of $P$. More precisely, if the order of $P$ is $n$ then $\partial P / \partial y^{(n)}$ does not vanish at $u$. For $\partial P / \partial y^{(n)}$ is obviously nonzero and of lower $s$-denomination than $P$.

\section{The approximation theorem.}

TheOREm. Let $\mathcal{F}$ be a nontrivial valued differential field, let $\mathrm{Z}$ be a nonzero differential subring of $\mathcal{F}$ such that $|a| \geqq 1$ for every nonzero element $a \in \mathrm{Z}$, let $u \in \mathcal{F}$ be differentially algebraic over $\mathrm{Z}$, let $s$ be any natural number $\geqq 1$, and denote the $s$-denomination of $u$ over $\mathrm{Z}$ by $d_{s}$. Then there exists an element $\gamma$ of the value group such that

$$
\left|u-a / b^{s}\right| \geqq \gamma /|b|^{d_{s}}
$$

for all elements $a, b \in \mathrm{Z}$ with $b \neq 0$ and $u \neq a / b^{s}$.

Proof. Let $P \in \mathcal{Z}\{y\}$ vanish at $u$ and have $s$-denomination $d_{s}$. By Lemma 4 there is an element $\gamma_{0}$ of the value group such that $|u-v| \geqq \gamma_{0}$ for every zero $v$ of $P$ with $v \in \mathcal{F}$ and $v \neq u$; we obviously may suppose that $\gamma_{0}<1$. Since $P(u)=0$ we may write

$$
P(y)=\sum_{k} c_{k}(y-u)^{\left(i_{k 1}\right)} \cdots(y-u)^{\left(i_{k, h(k)}\right)},
$$

where $c_{k} \in \mathcal{F}$ and $h(k) \geqq 1$ for each $k$. Setting

$$
\gamma_{1}=\sup _{k}\left|c_{k}\right| \beta^{i_{k 1}+\cdots+i_{k, h(k)}},
$$

we therefore see (using the first part of Lemma 1) that if $\left|u-a / b^{s}\right|$ $<\gamma_{0}$ then $\left|P\left(a / b^{s}\right)\right| \leqq \gamma_{1}\left|a / b^{s}-u\right|$ and $P\left(a / b^{s}\right) \neq 0 ;$ as $b^{d_{s}} P\left(a / b^{s}\right) \in \mathrm{Z}$ we have $\left|b^{d_{s}} P\left(a / b^{s}\right)\right| \geqq 1$, so that $\left|a / b^{s}-u\right| \geqq \gamma_{1}^{-1} /|b|^{d_{s}}$. On the other hand, if $\left|u-a / b^{s}\right| \geqq \gamma_{0}$ then obviously $\left|u-a / b^{s}\right| \geqq \gamma_{0} /|b|^{d_{s}}$. Setting $\gamma=\inf \left(\gamma_{0}, \gamma_{1}^{-1}\right)$, we find the theorem proved.

Columbia University 